\title{
Acute pulmonary edema and unstable coronary disease
}

Keywords: pulmonary edema, cardiogenic shock, aortic stenosis, pathophysiological, myocardial necrosis

Abbreviations: APE, acute pulmonary edema; HF, heart failure; ACS, acute coronary syndrome; CAD, coronary artery disease; AMI, acute myocardial infarction; ECG, electrocardiogram

\section{Opinion}

Acute pulmonary edema (APE) is one of the largest medical emergencies and it is the maximum expression of acute heart failure (HF). The current classification of acute HF recommended by the European Society of Cardiology takes into account the value of systolic pressure at admission: if the systolic pressure is $>140 \mathrm{mmHg}$, it is called high-pressure acute HF; between 90 and $140 \mathrm{mmHg}$, acute pressure-preserved HF; and $<90 \mathrm{mmHg}$, acute HF with hypotension. ${ }^{1}$

The 30-day mortality of the APE remains high. In 216 consecutive patients admitted with PAD in a single center, mortality was $14.1 \%{ }^{2}$ Among the modalities of acute cardiac insufficiency, it occupies the second place in hospital cost, the cardiogenic shock is the first one. ${ }^{3}$ Breathless is the main symptom of HF and in APE is usually very intense. The differential diagnosis of dyspnea in the emergency room is broad, but the main diseases to be excluded due to the severity and risk of death are $\mathrm{HF}$, acute coronary syndrome (ACS) and pulmonary embolism. ${ }^{4}$

In cardiogenic APE, the causes are, in particular, the diseases affecting the left heart, namely, systemic arterial hypertension, ischemic heart disease, aortic stenosis, mitral valvopathy cardiomyopathy and, in non cardiogenic, pneumonia and sepsis, trauma, bronchoaspiration and transfusion-related acute lung injury (TRALI). The exact mechanism of sudden increase in pulmonary capillary pressure in the cardiogenic APE is poorly understood, but the pathophysiological factor admitted is the loss of myocardial compliance, and the main cause attributed to this loss is myocardial ischemia with or without an acute ischemic event, causing ventricular dysfunction left. ${ }^{5}$

The most frequent anatomical substrate as cause of adult HF is coronary artery disease (CAD). Ischemic heart disease was classified as an occlusion of coronary disease greater than $75 \%$ in two epicardial arteries, or proximal lesion of the anterior descending or left main. In addition to the anatomical description, the history of myocardial revascularization or acute myocardial infarction entered the definition of ischemic heart disease. The parameter taken into consideration was the mortality curve between the coronary anatomy and other conditions known to cause heart failure. ${ }^{6}$

Severe CAD is often the anatomical substrate in patients with APE, as demonstrated by Graham at all in a study of 119 patients, of whom $71(60 \%)$ had a significant coronary lesion; 35 (49\%), lesion of three epicardial vessels; and $7(10 \%)$, left coronary trunk lesion. ${ }^{7}$

Acute HF occurred in only $13 \%$ of patients with an acute coronary syndrome (ACS), as demonstrated in the GRACE registry, ${ }^{8}$ which took into account for this diagnosis the presence of symptoms consistent with acute ischemia and at least one of the parameters:

Volume 2 Issue I - 2018

\author{
Maria das Neves Dantas da Silveira Barros,' \\ Nestor Rodrigues de Oliveira Neto ${ }^{2}$ \\ 'Pronto-Socorro Cardiológico de Pernambuco, Pernambuco \\ University, Brazil \\ ${ }^{2}$ Hospital Universitário Onofre Lopes, Federal University of Rio \\ Grande do Norte, Brazil
}

Correspondence: Maria das Neves Dantas da Silveira Barros, Pronto-Socorro Cardiológico de Pernambuco, Pernambuco University, Av. Agamenon Magalhães, S/N - Santo Amaro, Recife -PE, 50100-0I0, Brazil, Email mndantas@hotmail.com.br

Received: February 10, 2018 | Published: February 20, 2018

\section{i. ECG with acute ischemic alteration. \\ ii. Progressive increase of cardiac markers. \\ iii. Documentation of coronary disease.}

The careful evaluation for the identification of ischemia in the ECG is necessary, because the elevation of troponin, a biomarker of myocardial injury, occurs in several acute conditions, including HF, without considering the etiology. Therefore, this marker is not reliable for the diagnosis of ACS during the period of EAP. ${ }^{9}$

Several other cardiac conditions, in addition to acute myocardial infarction (AMI), and noncardiac ones may cause ST segment deviation, such as: pericarditis, left ventricular hypertrophy and left bundle branch block, Brugada syndrome and the early repolarization pattern. Left ventricular hypertrophy was the main confounding factor in the interpretation of ST elevation for the diagnosis of AMI. ${ }^{10}$ Despite the moderate sensitivity and specificity, the ECG is the main tool in the diagnosis of AMI with elevation of ST segment.

Electrocardiographic alterations - such as a marked inversion of the T wave and the increase in the QT interval - occurred during the APE, without representing an acute coronary event, in which the obstructive disease was removed, as evaluated in a series of cases with 9 patients. ${ }^{11}$

The electrocardiogram (ECG) is not able to provide the etiologic diagnosis of HF, but may suggest the cause of the heart failure and / or the triggering factor. Atrial fibrillation is the electrocardiographic finding with the highest likelihood ratio (LR), or a positive probability ratio for this diagnosis, its value being 3.8 (95\% CI 1.7-8.8), followed by new $\mathrm{T}$ wave changes, $\mathrm{LR}=3.0(95 \% \mathrm{CI} 1.7-5.3){ }^{12}$

Some authors have defined APE with positive myocardial necrosis markers as equivalent to non-ST elevation ACS, since creatine kinase - MB fraction rises to twice or more than the normal reference value, with or without ECG change. ${ }^{2,13}$ This is probably one of the reasons that a positive myocardium necrosis markers in PAD is attributed to an acute coronary event. 
As previously mentioned, elevation of troponin and electrocardiographic alteration suggestive of myocardial ischemia may occur in other cardiac conditions; therefore its usefulness in discriminating the patient with stable and unstable coronary disease may not be useful.

We carried out a study with 149 consecutive patients in a single referral center in the treatment of cardiovascular diseases with APE of uncertain etiology. Patients with electrocardiogram compatible with acute myocardial infarction with ST segment elevation were excluded. In the multivariate model, troponin elevation and electrocardiogram abnormalities such as dynamic ST and dynamic negative T were not able to discriminate patients with obstructive coronary artery disease (CAD) in an epicardial vessel in coronary angiography of patients without coronary artery disease. ${ }^{16}$ In this study, CAD was present in $59 \%$ of the sample.

Chest pain preceding the symptoms of heart failure was suggested by Kociol et al. ${ }^{14}$ to differentiate acute HF from acute coronary syndrome. In our study its presence preceding the episode of APE, as well as elevated levels of pro-BNP were also not helpful of discriminating non obstructive coronary disease. ${ }^{15}$

Therefore, the diagnosis of AMI is misleading because of the lack of specificity of the clinical picture, changes in the electrocardiogram and troponin. Considering dyspnea as a presentation of myocardial ischemia, as recorded by Grace $^{8}$ and Thygesen et al., ${ }^{16}$ and recognizing that chest pain may be an expression of other acute cardiac diseases, it is seen that the definition of APE precipitated by an unstable atherosclerotic plaque is an unlikely event, except in the myocardium infarct with ST elevation, where the ECG has a characteristic evolution. Thus, electrocardiographic alterations such as ST segment depression or T negative, increased troponin and chest pain may occur in the absence of acute obstructive coronary disease in the APE scenario.

\section{Acknowledgements}

None.

\section{Conflict of interest}

The author declares no conflict of interest.

\section{References}

1. Ponikowski P, Voors AA, Anker SD, et al. ESC Guidelines for the diagnosis and treatment of acute and chronic heart failure The Task Force for the diagnosis and treatment of acute and chronic heart failure of the European Society of Cardiology (ESC). Eur Heart J. 2016;37(27):2129 2200.
2. Figueras J, Peña-Gil C, Soler-Soler J. Thirty day prognosis of patients with acute pulmonary oedema complicating acute coronary syndromes. Heart. 2005;91(7):889-893.

3. Harjola VP, Costa S, Sund R, et al. The type of acute heart failure and the costs of hospitalization. Int J Cardiol. 2010;145(1):103-105.

4. Singer AJ, Thode HC, Green GB, et al. The incremental benefit of a shortness-of-breath biomarker panel in emergency department patients with dyspnea. Acad Emerg Med. 2009;16(6):488-494.

5. Ware LB, Matthay MA. Clinical practice: Acute Pulmonary Edema. $N$ Engl J Med. 2005;353(26):2788-2796.

6. Felker GM, Shaw LK, Oconnor CM. A Standardized Definition of Ischemic Cardiomyopathy for Use in Clinical Research. $\mathrm{J}$ Am Coll Cardiol. 2002;39(2):210-218.

7. Graham SP, Vetrovec GW. Comparison of angiographic findings and demographic variables in patients with coronary artery disease presenting with acute pulmonary edema versus those presenting with chest pain. Am J Cardiol. 1991;68(17):1614-1618.

8. Rationale and design of the GRACE (Global Registry of Acute Coronary Events) Project: A multinational registry of patients hospitalized with acute coronary syndromes. Am Heart J. 2001;141(2):190-199.

9. Felker GM, Teerlink JR. In: Mann DL, Douglas PZ, editors. Braunwald's Heart Disease. 10th ed. Elsevier, 2015. p. 484-511.

10. Wang K, Asinger RW, Marriott HJL. ST-Segment Elevation in Conditions Other Than Acute Myocardial Infarction. $N$ Eng $J$ Med. 2006;349(22):2128-2135.

11. Littmann L. Large $\mathrm{T}$ wave inversion and QT prolongation associated with pulmonary edema: a report of nine cases. J Am Coll Cardiol. 1999;34(4):1106-1110.

12. Wang CS, FitzGerald JM, Schulzer M, et al. Does this dyspneic patient in the emergency department have congestive heart failure? Jama. 2005;294(15):1944-1956.

13. Pena-Gil C, Figueras J, Soler-Soler J. Acute cardiogenic pulmonary edema. Relevance of multivessel disease, conduction abnormalities and silent ischemia. Int J Cardiol. 2005;103(1):59-66.

14. Kociol RD, Pang PS, Gheorghiade M, et al. Troponin elevation in heart failure: Prevalence, mechanisms, and clinical implications. $\mathrm{J}$ Am Coll Cardiol. 2010;56(14):1071-1078.

15. Barros. Predictors of Coronary Artery Obstructive Disease in Acute Pulmonary Edema of Unclear Origin. IJCS. 2018.

16. Thygesen K, Alpert JS, Jaffe AS, et al. Third universal definition of myocardial infarction. Eur Heart J. 2012;33:2551-2567. 